\title{
Theoretical modeling of mechanical homeostasis of a mammalian cell under gravity-directed vector
}

\author{
Lüwen Zhou $^{1,2}$ - Chen Zhang ${ }^{1,2}$ - Fan Zhang ${ }^{1,2}$ - Shouqin Lï ${ }^{1,2}$ - Shujin Sun ${ }^{1,2}$. \\ Dongyuan L $\ddot{\ddot{u}}^{1,2}$. Mian Long ${ }^{1,2}$ (1)
}

Received: 16 February 2017 / Accepted: 8 August 2017 / Published online: 17 August 2017

(C) Springer-Verlag GmbH Germany 2017

\begin{abstract}
Translocation of dense nucleus along gravity vector initiates mechanical remodeling of a eukaryotic cell. In our previous experiments, we quantified the impact of gravity vector on cell remodeling by placing an MC3T3-E1 cell onto upward $(U)$-, downward $(D)$-, or edge-on $(E)$ - orientated substrate. Our experimental data demonstrate that orientation dependence of nucleus longitudinal translocation is positively correlated with cytoskeletal (CSK) remodeling of their expressions and structures and also is associated with rearrangement of focal adhesion complex (FAC). However, the underlying mechanism how CSK network and FACs are reorganized in a mammalian cell remains unclear. In this paper, we developed a theoretical biomechanical model to integrate the mechanosensing of nucleus translocation with CSK remodeling and FAC reorganization induced by a gravity vector. The cell was simplified as a nucleated tensegrity structure in the model. The cell and CSK filaments were considered to be symmetrical. All elements of CSK filaments and cytomembrane that support the nucleus were simplified as springs. FACs were simplified as an adhesion cluster of parallel bonds with shared force. Our model proposed that gravity vector-directed translocation of the cell nucleus is mechanically balanced by CSK remodeling and FAC reorganization induced by a gravitational force. Under gravity, dense nucleus tends to translocate and exert additional com-
\end{abstract}

Mian Long

mlong@imech.ac.cn

1 Center for Biomechanics and Bioengineering, Key Laboratory of Microgravity (National Microgravity Laboratory), and Beijing Key Laboratory of Engineered Construction and Mechanobiology, Institute of Mechanics, Chinese Academy of Sciences, Beijing, China

2 School of Engineering Science, University of Chinese Academy of Sciences, Beijing, China pressive or stretching force on the cytoskeleton. Finally, changes of the tension force acting on talin by microfilament alter the size of FACs. Results from our model are in qualitative agreement with those from experiments.

Keywords Gravity directed · Mechanosensing · Nucleus translocation $\cdot$ Cytoskeletal remodeling $\cdot$ FAC reorganization

\section{Introduction}

There is much evidence to show that single cells in vitro respond to changes in gravity and this response plays a major role in physiological changes at the organism level (Vorselen et al. 2014). Gravity variations can lead to changes in cell proliferation, differentiation, intracellular signaling, and gene expression (Clément and Slenzka 2006; Nichols et al. 2006; Grimm et al. 2011; Cogoli and Cogoli-Greuter 1996). Recent studies have focused on the molecular mechanism behind the perception of cellular mechanical microenvironment and the subsequent mechanotransduction to biochemical signals, ultimately leading to changes in cell function (Orr et al. 2006; Geiger et al. 2009). However, cellular responses to mechanical stimuli are far from being completely understood.

Cells on Earth experience multiple mechanical forces including gravity. These forces or stresses often require structural restabilization mainly originated from cytoskeletal (CSK) remodeling, which plays a role similar to the structural scaffold of a building. Unlike a concrete building, gravitational forces are usually ignored in a single cell, because cellular gravity is presented at a small geometric scale, a low Reynolds number, and a low density differences between the organelles (i.e., mitochondria or nuclei) and the cytosol (Feric and Brangwynne 2013). Although 
the force generated by polymerization of microtubule and actin or by a single molecular motor (in the order of several pNs) is almost ten times higher than that exerted on a mammalian cell by gravity (in the order of $0.5 \mathrm{pN}$ ), it is inappropriate to exclude the effect of gravity directly. This is because the integrated effect of specific organelle sedimentation, which is attributed to the density difference between organelles and cytosol, may not be negligible for a longtime adherence onto the surface. Recent works on large $X$. laevis oocytes (Feric and Brangwynne 2013; Feric et al. 2015) support this viewpoint to some extent. It was found that the gravity is dominant and encountered by a nuclear actin network that prevents nuclear bodies from immediate sedimentation and coalescence in large $X$. laevis oocytes (Feric and Brangwynne 2013). However, the actin network is weak, which could not indefinitely support the emulsion of nuclear bodies, but, instead, only stabilizes them by slowing down gravitational creep up to two months (Feric et al. 2015).

Recently, we developed an orientation-varied assay to quantify the impact of gravity vector on mechanical stability and biological phenotype of a typical mammalian cell type of MC3T3-E1 cells. By placing the cells onto an upward $(U)-$, downward $(D)$-, or edge-on $(E)$-orientated substrate, longtime nucleus longitudinal translocation initiates the CSK remodeling to resist the translocation mechanically and then mediate the focal adhesion complex (FAC) reorganization to anchor the cell stably on the substrate (Zhang et al. 2017). This orientation-varied approach is based on the strategy that vector-directed gravity varies in a constant angle, rather than that changed periodically in clinostat (Vorselen et al. 2014), with respect to the culture substrate, which brings up an opportunity to conduct theoretical analyses on how the cytoskeleton itself is affected and how the CSK network is reorganized to maintain mechanical homeostasis on altered gravity vector in long time.

Previous mechanical models of single cells mainly include continuous models and structural models. Continuum cell models, treating the cell as comprising materials with certain continuum material properties (Lim et al. 2006), provide fewer insights into the detailed molecular mechanical events. Structural models, accounting for the overall cellular behaviors as a result of the collective physicochemical interactions between structures forming the cytoskeletons (Nava et al. 2014), mainly include cable network models (Stamenović and Coughlin 1999), cell foams models (Satcher and Dewey 1996), and tensegrity models (Ingber 1993). In this work, we developed a mechanical model based on nucleated tensegrity structure to specify the mechanical balance for three CSK elements of microfilament, microtubule, and intermediate filament and FACs inside a cell. Our model also provided the predictions on the contributions of FAC formation and the damping effects of the cytoskeletons.

\section{Experiments}

Mammalian cells with a normal size $(\sim 10 \mu \mathrm{m}$ in diameter) can sense the alteration of gravity vector (Vorselen et al. 2014). In our previous experiments, mouse MC-3T3E1 cells are placed onto $U$-(Fig. 1a), $D$-(Fig. 1b), or $E$-orientated (Fig. 1c) substrate for 24 or $72 \mathrm{~h}$ (Zhang et al. 2017). Notably, the impact of hydrostatic pressure on orientation dependence of cellular responses is excluded due to the minimal pressure differences in the three orientations ( $\mathrm{Li}$ et al. 2010). Collected cells at given time points are then stained with fluorescent antibodies to actin, tubulin, and vimentin for testing the orientation dependence of CSK proteins. The nucleus is stained for measuring its translocation inside the cell. Stained actin is also used to identify the contour of the cell for morphological analysis. Typically, no significant differences are found for cell projected area, circularity, and aspect ratio in three orientations at $24 \mathrm{~h}$ (Zhang et al. 2017), indicating that the cells placed onto differently orientated substrates can insist their mechanical stability and there is no difference between cellular morphologies in three orientations. We further measured the cell radius (Fig. 1d) and thickness (Fig. 1e) as well as nucleus radius (Fig. 1f) and volume (Fig. 1h) using 3D reconstruction of the sliced fluorescent images. Not only these measurements support the previous observations, but they will also be used as input parameters for our modeling below.

Gravity is able to mechanically direct the nucleus sedimentation and induces the cell and CSK remodeling. Cells placed onto different orientated substrates could mediate discrepant translocation of the dense nucleus in cytosol on Earth's gravity. Here we replotted the data of nucleus longitudinal height from Zhang et al. (2017), which reads $\sim 1 / 2$ (Fig. 1g) of the typical cell height (Fig. 1e) with less scattered distribution. The orientation dependence of the height is observed, which is higher in $D$ compared with those in $U$ or $E$, implying that the nucleus longitudinal translocation behaves differently in these orientated substrates.

CSK remodeling provides the internal forces to resist the nucleus sedimentation. Here mechanical stability of gravity vector-directed nucleus translocation is reached by the balance between the net force of nucleus gravity minus buoyant force and mechanical forces exerted by CSK network. This is observed by the higher expressions of global actin or vimentin in $D$ than those in $U$ or $E$ (Zhang et al. 2017), which is positively correlated with the nucleus longitudinal height (cf., Fig. 1g) and predicts that the mechanical stability of nucleus in cytosol is mainly supported by actin and vimentin at $24 \mathrm{~h}$. To isolate the respective contributions of these CSK filaments localized distinctly inside a cell, we further analyzed the regional expression of CSK proteins by segregating the cell into apical and basolateral halves (inserts in Fig. 2a-c). Data indicated that all the three elements present lower expression in $D$ than those in $U$ or $E$ in 

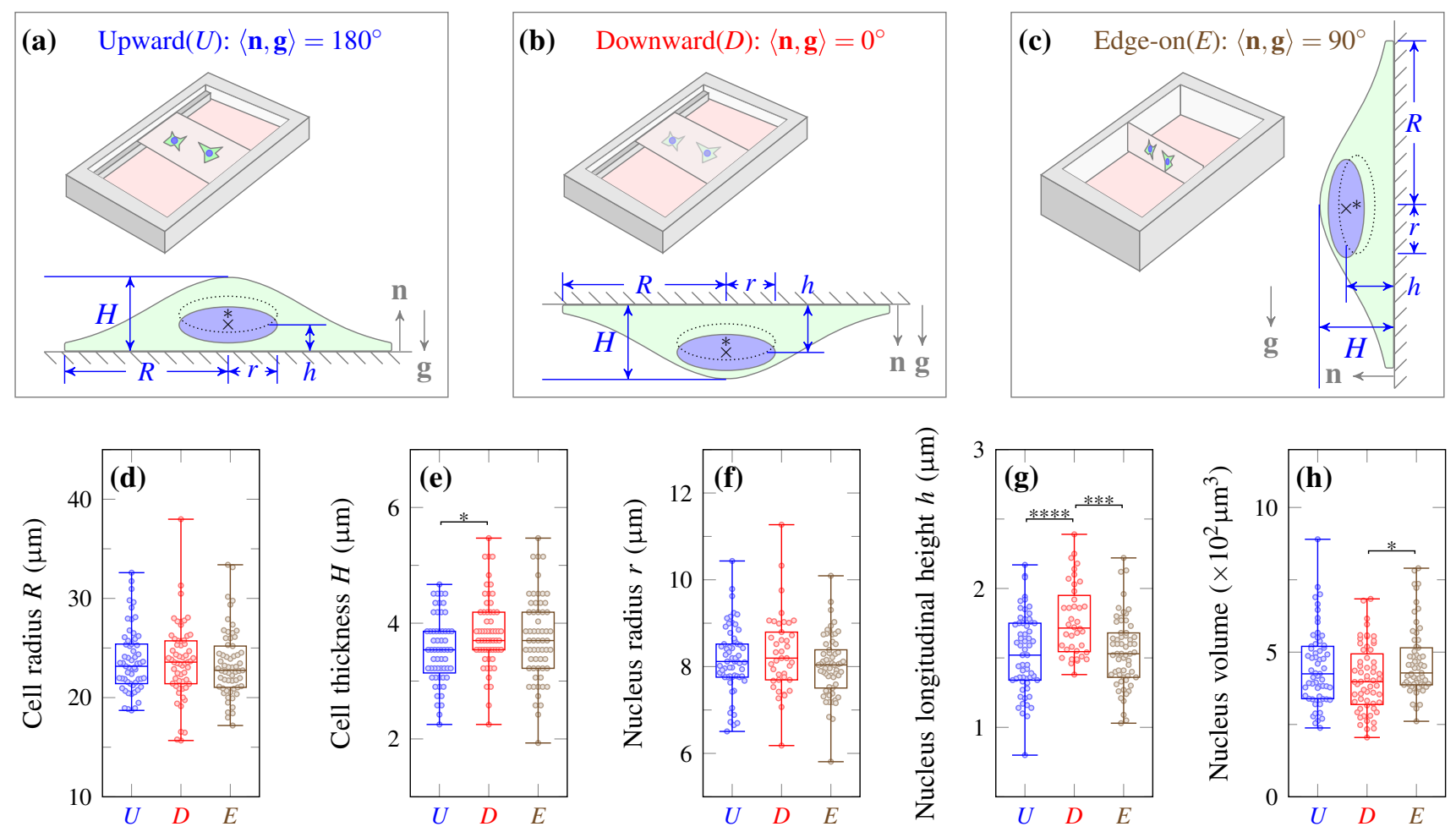

Fig. 1 a-c Schematic of MC3T3-E1 cells cultured in upward a downward $\mathbf{b}$ or edge-on textbfc oriented substrate. $\mathbf{d}-\mathbf{h}$ Cell and nucleus morphology. Here cell radius $R$ and thickness $H$ as well as nucleus radius $r$ and longitudinal height $h$ are defined in the respective panels $\mathbf{a}-\mathbf{c}$, in which $h$ is defined as the distance from nucleus centroid to the substrate (nucleus contour is depicted as a solid or dotted line; asterisk

the apical half, suggesting that the cells placed in $D$ unlikely enrich these CSK filaments in the apical half. Specifically, the apical fraction is $\leq 0.5$ for actin or tubulin (Fig. 2a, b) or $\geq 0.5$ for vimentin (Fig. $2 \mathrm{c}$ ) in three orientations, indicating that actin and tubulin tend to be enriched in the basolateral half, but vimentin is likely localized in the apical half of the cell. Noting that longitudinal direction in $E$ is perpendicular to gravity vector, we then determined the regional distributions of the cytoskeletons opposite to or along with gravity vector. Here this specialized fraction value in $E$ all yielded $\sim 0.5$ for actin, tubulin, and vimentin (most right bars in Fig. 2a-c), implying the irrelevance of CSK remodeling in $E$ with the direction of gravity vector. Taken together, all the three cytoskeletons tend to be accumulated in the apical half in $D$, which is reversely correlated with the nucleus longitudinal height.

FAC formation is regulated by CSK remodeling since FACs serve as major mechanical elements connecting the intracellular structures to the outer substrate. Thus, we tested the orientation dependence of FAC depicted as colocalized actin, vinculin, paxillin, and $\beta 1$-integrin (Fig. 3a, only actin and vinculin are shown). Without losing generality, we compared the regional distribution for cells placed in

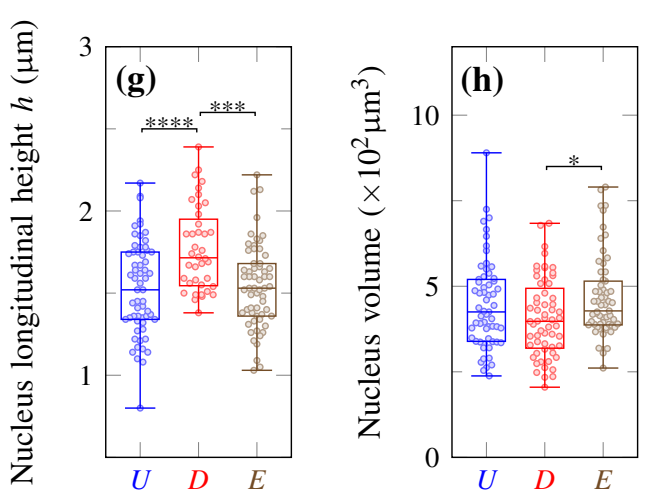

denotes cell centroid). Cell radius (d) and thickness (e), nucleus radius (f) and longitudinal height (g), as well as nucleus volume (h) at $24 \mathrm{~h}$ are plotted in three orientations. The equivalent radius of cell or nucleus is approximated by the square root of the projected area divided by $\pi$. Data for nucleus longitudinal height are adopted from Zhang et al. (2017). $* p<0.05$; *** $p<0.001 ; * * * * p<0.0001$

$E$-orientation at $24 \mathrm{~h}$. Again, by segregating the cell in $E$ into two regions opposite to $(y>0)$ or along with gravity director $(y<0)$, FAC localized distribution presented higher FAC area but similar FAC number (Fig. 3b). Since mechanical strength of cell anchorage is mainly determined by FAC area, these data indicated the differential contributions of localized FACs in supporting cellular mechanical stability in $E$. Meanwhile, no differences of localized FACs in the left and right regions at the plane parallel to the substrate were found for the cells in $U$ or $D$ (data not shown), as expected. Thus, these analyses provided the base for our mathematical modeling below.

\section{Models and results}

\subsection{Gravity vector-directed translocation of the cell nucleus is deterministic}

Organelles, such as nucleus and mitochondria with a relatively large size, are often of higher density than the cytosol. Earlier theoretical analysis (Pollard 1965) indicated that the nucleus might be sufficiently large and dense to sedi- 

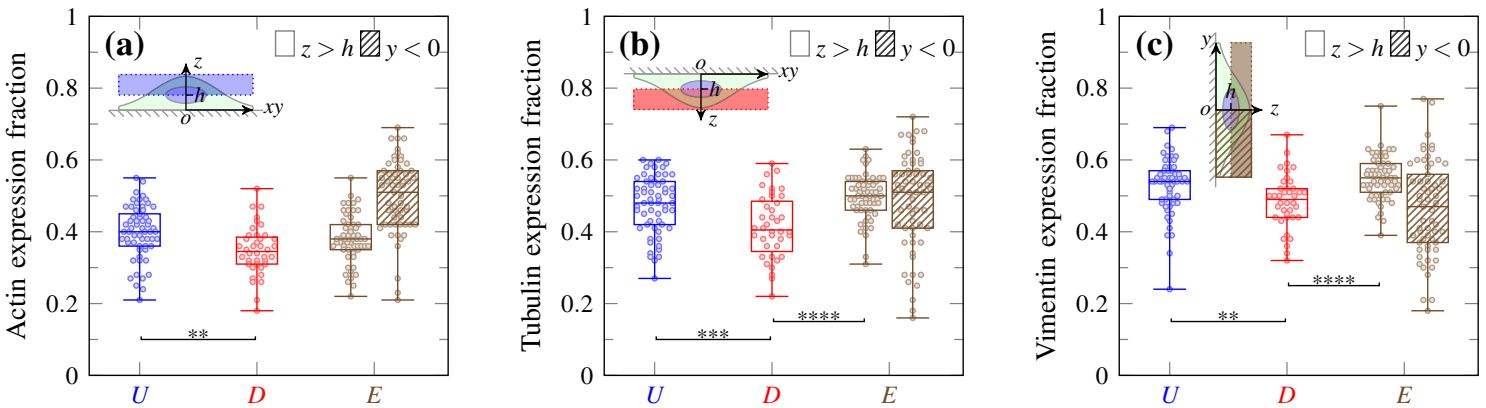

Fig. 2 Expressions of apical actin (a), tubulin (b), and vimentin (c) of a cell in three orientations. Data are defined as the fraction of the CSK element in the apical half (shading region separated by the plane at the cell centroid and parallel to the substrate, as indicated in each insert) in whole cell. Also defined is the regional distribution of CSK

elements along gravity vector in $E$, where the fraction in the half region opposite to gravity vector (separated by the plane at the cell centroid and perpendicular to the substrate) in whole cell is calculated (c). ** $p<0.01 ; * * * p<0.001$; **** $p<0.0001$
Fig. 3 Localized distribution of FACs in edge-on orientation. a The typical image is illustrated for the definition of gravity vector-related distribution in two regions $(y>0$ and $y<0$ opposite to and along with gravity vector, respectively) in $E$ - orientation. (red: vinculin, green: actin, blue: nucleus) b The regional FAC number and area in $E$-orientation at $24 \mathrm{~h}$ are presented as median values with 25 and 75 th percentiles of 4060 cells from three repeated experiments
Table 1 Model parameters of single cell
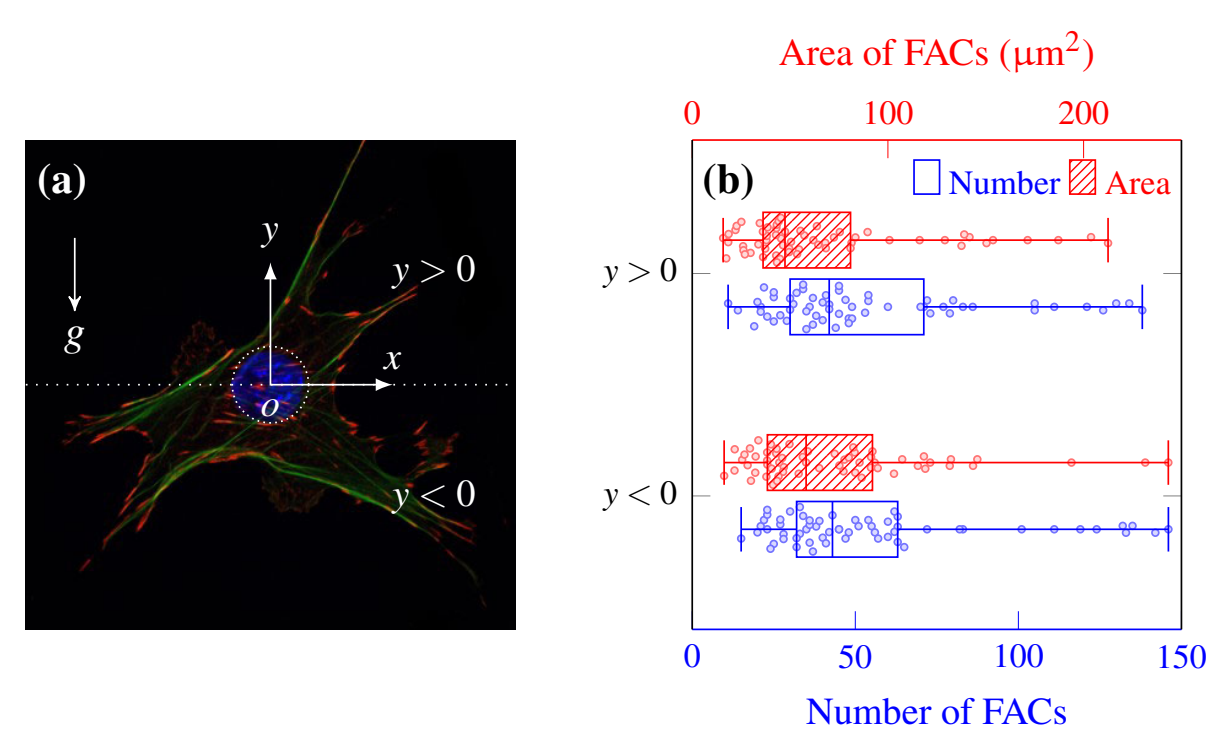

\begin{tabular}{lllll}
\hline Size scale & Symbol & Definition & Value & Source \\
\hline Cell & $H$ & Thickness of cell & $4 \mu \mathrm{m}$ & Figure 1e \\
& $\rho$ & Density of cytosol & $1.1 \mathrm{~g} / \mathrm{ml}$ & Wegener et al. (1998) \\
& $\mu$ & Viscosity of cytosol & $2 \times 10^{-2} \mathrm{~Pa} \mathrm{~s}$ & Kalwarczyk et al. (2011) \\
Subcell & $V$ & Volume of nucleus & $500 \mu \mathrm{m}^{3}$ & Figure 1h \\
& $\rho$ & Density of nucleus & $1.4 \mathrm{~g} / \mathrm{ml}$ & George et al. (2011) \\
\hline
\end{tabular}

ment under the influence of gravity if the nucleus could be considered as a solid object suspended in a viscous liquid medium.

Table 1 summarizes the parameters of single typical osteoblast in our experiments. For a nucleus with volume $500 \mu^{3}$ and density $1.4 \mathrm{~g} / \mathrm{ml}$, the apparent weight in cytosol with density $1.1 \mathrm{~g} / \mathrm{ml}$ is approximately equal to $1.5 \mathrm{pN}$. Comparing the gravitational energy of a weight of $1.5 \mathrm{pN}$ at an average distance of the half height $(2 \mu \mathrm{m})$ above the lowest point of the cell with thermal energy associated with diffusive motion, we have

$G_{T}=\frac{\Delta \rho V g H / 2}{k_{\mathrm{B}} T} \sim O\left(10^{3}\right) \gg 1$.

Here $\Delta \rho$ is the density difference between nucleus and cytosol, $V=$ is the nucleus volume, $g$ is gravity acceleration, $H$ is the cell thickness, $k_{\mathrm{B}}$ is Boltzmann constant, and $T=300 \mathrm{~K}$ is absolute temperature. The dimensionless 
number $G_{T}$ represents the competitive relationship between gravitational sedimentation and Brownian motion. $G_{T} \gg 1$ indicated that gravity effects plays a absolute dominant role over random fluctuation in the directional movement of nucleus. Thus, if there is a directed translocation of nucleus, it must be caused by gravity.

Furthermore, we can also estimate the sedimentation velocity of the nucleus if we were to approximate the nucleus as a sphere $r=5 \mu \mathrm{m}$ in radius (the corresponding vol-

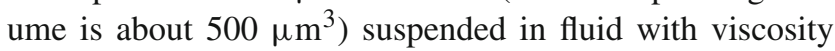
$\mu=2 \times 10^{-2} \mathrm{Pas}$ (this value is the same as viscosity of cytosol). Considering the forces acting on the sphere: $m \partial^{2} x / \partial t^{2}=F_{\text {drag }}-F_{\mathrm{g}}$, where $F_{\text {drag }}=6 \pi \mu r v$ is the drag force, and $F_{g}=4 / 3 \pi r^{3} \Delta \rho g$ is the net force of nucleus gravity minus buoyant force. At steady state, the acceleration is zero, and one obtains:

$v=\frac{2 r^{2} \Delta \rho g}{9 \mu} \approx 8 \times 10^{-7} \mathrm{~m} / \mathrm{s}$.

It is obvious that an additional stabilization mechanism is necessary; otherwise, all nuclei will sediment to the bottoms of their cells with a few seconds under Earth's gravity.

\subsection{Directed nucleus translocation is mechanically balanced by CSK reorganization}

It was obvious that the discussion of sedimentation velocity above did not yet consider a structural framework (e.g., the cytoskeleton) in its approximation. Experiments show that if cultured cells on coverslips were treated with cytochalasin B and then subjected to a centrifugal field, the acceleration is then adequate to enucleate the cells (Prescott et al. 1972). It is indicated that gravitational sedimentation does not happen as long as CSK filaments provide a stabilizing scaffold for nuclei.

The cytoskeleton can perform a multitude of functions. Primarily, it supports the cell shape and mechanical resistance to deformation, so that through association with extracellular connective tissue and other cells, it stabilizes entire tissues (Herrmann et al. 2007). The cytoskeleton can also contract actively, thereby deforming the cell, altering the cell's environment, and allowing the cell to migrate (Fletcher and Mullins 2010). Eukaryotic cells contain three main kinds of CSK filaments: microfilaments (actin filaments), microtubules, and intermediate filaments. Each CSK filament has a shape and intracellular distribution. Previous studies (Zhang et al. 2017; Vorselen et al. 2014) have indicated that three main kinds of CSK filaments contain following features:

- Microfilaments (MFs): MFs are fine, thread-like protein fibers, 6-8 $\mathrm{nm}$ in diameter. They are composed predominantly of a contractile protein called actin, which is the most abundant cellular protein. MFs' association with the protein myosin is responsible for muscle contraction. In an adherent cell, most MFs have a radial organization and distribute uniformly. MFs mainly bear tension in the maintenance of cell's shape.

- Intermediate filaments (IFs): IFs, averaging $10 \mathrm{~nm}$ in diameter, are more stable (strongly bound) than actin filaments and are heterogeneous constituents of the cytoskeleton. IFs organize the internal three-dimensional structure of the cell, anchoring organelles and serving as structural components of the nuclear lamina. They are located at the vicinity of the nucleus to form the perinuclear rings (Kamei 1994). Like actin filaments, IFs' function in the maintenance of cell shape by bearing tension.

- Microtubules (MTs): MTs are cylindrical tubes, about $20 \mathrm{~nm}$ in diameter. They are composed of subunits of the protein tubulin termed $\alpha$ and $\beta$. In an adherent cell, MTs often have a radial organization and express more perinuclearly. MTs mainly resist compression in the maintenance of cell's shape.

In the light of the above features of CSK filaments and drawing on the basis of previous work (Wang et al. 2009), a nucleated tensegrity structure, as shown in Fig. 4a, is proposed to characterize CSK structure. Local forces applied to integrins by ECM are concentrated at focal adhesions (FAs) and transmitted to MFs, which is bundled by $\alpha$-actinin and made tensed by myosin II to generate prestress. MFs are connected to MTs through MACF1 and to IFs through plectin. Plectin also connects IFs with MTs and IFs with nesprin on the outer nuclear membrane. Nesprins connect MFs to the inner nuclear membrane protein SUN.

This CSK structure is able to reorganize for generating additional force, which can balance the net force of nucleus gravity minus buoyant force in a specific orientation. Figure 4b-d illustrates force analysis of cells in $U-, D$-, and $E$-oriented substrates (only CSK filaments which resist gravitational sedimentation of nucleus are drawn in the figure). In order to simplify the analysis, the cell and CSK filaments are considered to be symmetrical, and all elements for supporting nucleus, including CSK filaments and cytomembrane, are simplified as springs. Then, the length of MFs and MTs can be expressed as

$\ell \approx(R-r) / \cos \theta$

where $R$ is the cell radius, $r$ is the nucleus radius, and $\theta$ is the angle between MFs or MTs and substrates. It is easy to see that $\theta \approx h / R$ since $h \ll R$, where $h$ is the distance from nucleus centroid to the substrate surface. Next, we will consider the issue of estimating the length of nucleus translocation in three orientations, respectively. 


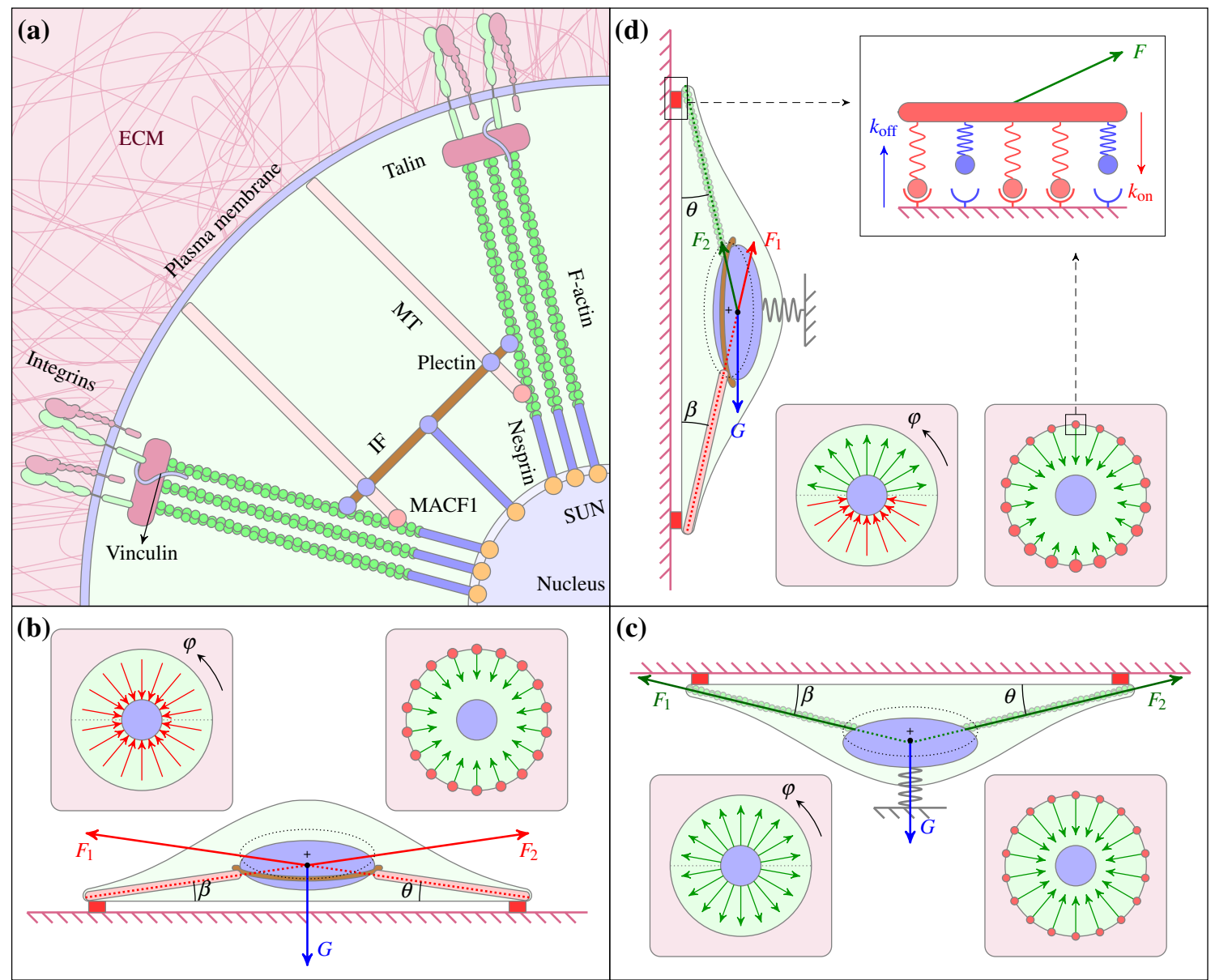

Fig. 4 A working model of gravity-directed remodeling for a mammalian cell in oriented substrate. a Mechanical stability of the dense nucleus is supported by CSK network. b-d Mechanical forces exerted on the nucleus are balanced differentially by tension-resistant actin, compression-resistant tubulin, and connecting vimentin in upward (b), downward (c), or edge-on orientation (d)
As shown in Fig. 4b, nucleus tends to migrate toward the substrate under gravity in $U$-orientation. Nucleus translocation enhances the compression of MTs and weakens the tension of MFs since MFs play the role of tension supporting structures. The nuclear radius $r$ decreases in $U$-orientation since nucleus is compressed in the direction parallel to the substrate. To prevent infinite translocation, MTs and IFs will be reorganized, against the nucleus translocation $\Delta h^{U}$ having already occurred. Our experiments showed that IFs is a more perinuclear distribution to form the perinuclear rings. Thus, it is easy for a nucleus to migrate in the direction perpendicular to IFs ring plane, but hard in the direction parallel to IFs ring plane. In this orientation, MTs spring and IFs spring are in series to support the nucleus. Therefore, $\Delta h^{U}$ can be divided into two parts, namely the displacement of nucleus relative to IFs, $\Delta h_{i}^{U}$, and the displacement of IFs relative to substrate, $\Delta h_{t}^{U}$. The first part displacement $\Delta h_{i}^{U}$ causes IFs' reorganization. Thus,
$G=n_{i} k_{i} \Delta h_{i}^{U}=K_{i} \Delta h_{i}^{U}$,

where $G$ is the apparent weight of nucleus in cytosol, $n_{i}$ is the number of IFs, $k_{i}$ is the spring coefficient of a single IF, and $K_{i}=n_{i} k_{i}$ reflects the equivalent spring constant of all IFs. The second part displacement $\Delta_{t}^{U}$ causes MTs' reorganization. The compressed length of a single MT can be estimated from

$$
\begin{aligned}
\Delta \ell_{t} & =\mathrm{d} \ell_{t}=\mathrm{d}\left(\frac{R-r}{\cos \theta}\right)=(R-r) \frac{\sin \theta}{\cos ^{2} \theta} \mathrm{d} \theta \\
& =(R-r) \frac{\sin \theta}{\cos ^{2} \theta} \frac{\mathrm{d} h}{R-r} \approx \theta \Delta h_{t}^{U} .
\end{aligned}
$$

All compression springs together offer an additional force to balance the apparent weight of nucleus,

$$
\begin{aligned}
G & =n_{t} F_{t} \sin \theta=n_{t} k_{t} \theta \mathrm{d} h_{t} \sin \theta \\
& =n_{t} k_{t} \theta^{2} \mathrm{~d} h_{t}=n_{t} k_{t} \frac{h^{2}}{R^{2}} \mathrm{~d} h_{t}=K_{t} \Delta h_{t}^{U},
\end{aligned}
$$


where $n_{t}$ is the number of MTs, $k_{t}$ is the spring coefficient of a single MF, and $K_{t}=n_{t} k_{t} h^{2} / R^{2}$ reflects the equivalent spring constant of all MTs. MTs spring and IFs spring are in series to together support nucleus; thus,

$$
\frac{K_{i} K_{t}}{K_{i}+K_{t}} \Delta h^{U}=G \rightarrow \Delta h^{U}=\frac{K_{i}+K_{t}}{K_{i} K_{t}} G \sim \frac{G}{K_{t}} .
$$

It should be noted that the above derivation assumes $K_{t} \ll$ $K_{i}$. The rationality of this assumption will be discussed later in this section.

As shown in Fig. 4c, nucleus tends to migrate apart from the substrate under gravity in $D$-orientation. Nucleus translocation enhances the tension of MFs and weakens the compression of MTs since MTs play the role of compression supporting structures. The nuclear radius $r$ increases in $D$-orientation since nucleus is stretched in the direction parallel to the substrate. To prevent infinite translocation, MFs are reorganized to mainly against the nucleus translocation $\Delta h^{D}$ having already occurred. By a similar analytical approach to that of Eq. (5), the stretched length of a single MF can be estimated as $\Delta \ell \approx \theta \Delta h^{D}$. All elongated springs together offer an additional force,

$F_{a}=n_{a} k_{a} \frac{h^{2}}{R^{2}} \mathrm{~d} h=K_{a} \Delta h^{D}$,

where $n_{a}$ is the number of MFs, $k_{a}$ is the spring coefficient of a single MF, and $K_{a}=n_{a} k_{a} h^{2} / R^{2}$ reflects the equivalent spring constant of all MFs. In this case, cytomembrane and CSK filaments between cytomembrane and nucleus may also play a role in supporting nucleus. Here, we also simplify cytomembrane and CSK filaments between cytomembrane and nucleus as a so-called membrane spring with coefficient $K_{\mathrm{m}}$. MFs spring and membrane spring are in parallel to support nucleus; thus,

$$
\left(K_{\mathrm{m}}+K_{a}\right) \Delta h^{D}=G \rightarrow \Delta h^{D}=\frac{G}{K_{\mathrm{m}}+K_{a}} \sim \frac{G}{K_{a}} .
$$

It should be noted that the above derivation assumes $K_{\mathrm{m}} \ll$ $K_{a}$. The rationality of this assumption will also be discussed later in this section.

As shown in Fig. 4d, the direction of gravity is approximately parallel to the plane of CSK filaments in $E$ orientation. This paralleling may lead to nucleus hard to migrate along gravity vector. Nucleus tends to migrate away from the substrate since compression of MTs prevents, via enhanced vimentin expression and ring formation, the nucleus translocation along gravity vector. To test this, nucleus translocation along gravity vector $\Delta h_{\|}$was estimated. If $\Delta h^{E}=\Delta h_{\|}$is parallel to gravity, the compressed length of a single MT will be (for the detailed derivation, see "Appendix 1")
$\Delta \ell_{t}=\mathrm{d} \ell_{t}=\sin \varphi \cos \beta \mathrm{d} h=\sin \varphi \cos \beta \Delta h_{\|}$.

We assume that MTs are uniform distribution on the angle $\varphi$. If the nucleus is supported only by MTs, thus,

$$
\begin{aligned}
G & =-\int_{\pi}^{2 \pi} \frac{n_{t} k_{t}}{2 \pi} \sin \varphi \cos \beta \Delta h_{\|} \cos \beta \mathrm{d} \varphi \\
& =-\frac{n_{t}}{2 \pi} \cos ^{2} \beta \Delta h_{\|} \int_{\pi}^{2 \pi} \sin \varphi \mathrm{d} \varphi \\
& =\frac{n_{t} k_{t}}{\pi} \cos ^{2} \beta \Delta h_{\|} \\
& \approx \frac{n_{t} k_{t}}{\pi} \Delta h_{\|} .
\end{aligned}
$$

From Eq. (11), $\Delta h_{\|}$can be obtained and compared with nucleus translocation in $U$ from Eq. (7):

$\Delta h_{\|} \approx \pi \frac{G}{n_{t} k_{t}}=\frac{\pi h^{2}}{R^{2}} \frac{1}{K_{t}} G \ll \Delta h^{U}=\frac{1}{K_{t}} G$

Equation (12) indicates that nucleus translocation along gravity vector in $E$ is negligible. Therefore, the length of MTs is considered as constant in this process, and the nucleus translocation may be perpendicular to MTs and thus approximately perpendicular to the substrate. If $\Delta h^{E}=\Delta h_{\perp}$ was perpendicular to MTs, the stretched length of a single MF will be (for the detailed derivation, see "Appendix 1")

$\Delta \ell_{a}=\sin \theta \cos \theta(1+\sin \varphi) \Delta h_{\perp}$.

Membrane spring could have an effect in this case. Then, to balance the torques, we have:

$$
\begin{aligned}
G h & =\frac{1}{2} n_{a} k_{a} \theta \Delta h_{\perp} h+n_{a} k_{a} \theta^{2} \Delta h_{\perp} R+K_{\mathrm{m}} \Delta h_{\perp} R \\
& =\frac{1}{2} K_{a} \Delta h_{\perp} R+K_{a} \Delta h_{\perp} R+K_{\mathrm{m}} \Delta h_{\perp} R \\
& =\left(\frac{3}{2} K_{a}+K_{\mathrm{m}}\right) \Delta h_{\perp} R .
\end{aligned}
$$

From Eq. (14), $\Delta h_{\perp}$ can be obtained and compared with $\Delta h_{\|}:$

$$
\begin{aligned}
\Delta h_{\perp} & =\frac{1}{3 K_{a} / 2+K_{\mathrm{m}}} \frac{h}{R} G \sim \frac{2}{3 K_{a}} \frac{h}{R} G \\
& \gg \frac{\pi h^{2}}{R^{2}} \frac{1}{K_{t}} \approx \Delta h_{\|} .
\end{aligned}
$$

It should be noted that the above derivation assumes $K_{a} \ll$ $K_{t} R^{2} / h^{2}$. The rationality of this assumption will be discussed later in this section. Equation (15) indicates that nucleus translocation is perpendicular to the substrate, which 
Table 2 Elastic properties of CSK filaments (Mofrad and Kamm 2006; Milo and Phillips 2015)

\begin{tabular}{llllrl}
\hline & Diameter $d(\mathrm{~nm})$ & Cross-sectional area $A\left(\mathrm{~nm}^{2}\right)$ & Persistence length $(\mu \mathrm{m})$ & Bending stiffness $\left(\mathrm{Nm}^{2}\right)$ & Yong's modulus $E(\mathrm{~Pa})$ \\
\hline MF & $6-8$ & 20 & 15 & $7 \times 10^{-26}$ & $1.3-2.5 \times 10^{9}$ \\
MT & 25 & 200 & 6000 & $2.6 \times 10^{-23}$ & $1.9 \times 10^{9}$ \\
IF & 10 & $\pi(d / 2)^{2} \approx 80$ & $\sim 1$ & $4 \times 10^{-27}$ & $1 \times 10^{9}$ \\
\hline
\end{tabular}

Table 3 Translocation and longitudinal height of nucleus in three orientations

\begin{tabular}{llllll}
\hline Orientation & Translocation & Equation & Comparison & Longitudinal height & Comparison \\
\hline$U$ & $\Delta h^{U} \sim G / K_{t}$ & $(7)$ & $\Delta h^{U} \ll \Delta h^{D}$ & $h^{U}=h_{0}-\Delta h^{U}$ & $h^{U}<h^{D}$ \\
$D$ & $\Delta h^{D} \sim G / K_{a}$ & $(9)$ & & $h^{D}=h_{0}+\Delta h^{D}$ \\
$E$ & $\Delta h^{E} \sim(h / R) \cdot G / K_{a}$ & $(16)$ & $\Delta h^{E} \ll \Delta h^{D}$ & $h^{E}=h_{0}+\Delta h^{E}$ & $h^{E}<h^{D}$ \\
\hline
\end{tabular}

Longitudinal height $h=h_{0}-\Delta h$ for nucleus migration toward the substrate and $h=h_{0}+\Delta h$ for nucleus migration apart from the substrate, where $h_{0}$ is the longitudinal height of nucleus at the initial time

is consistent with our previous experimental results (Zhang et al. 2017). Finally, we have

$\Delta h^{E} \approx \Delta h_{\perp}=\frac{1}{3 K_{a} / 2+K_{\mathrm{m}}} \frac{h}{R} G \sim \frac{h}{R} \frac{1}{K_{a}} G$.

As discussed above, nucleus translocation in three orientations can be obtained by establishing the statics equilibrium equations of the nucleus. It should be noted that our above derivation relies on specific assumptions about the relative strength of three springs. The relationship between Young's modulus and the spring constant is $k=Y A / \ell$, where $Y$ is Young's modulus, $A$ is the cross-sectional area, $\ell$ is the initial length. Table 2 summaries elastic properties of CSK filaments. Young's modulus of three CSK filaments is in the same order of magnitude $\left(Y_{t} \sim Y_{a} \sim Y_{i}\right)$. The differences among cross-sectional area of three CSK filaments are within one order of magnitude $\left(A_{a}<A_{i}<A_{t}\right.$ and $\left.A_{a} \ll A_{t}\right)$. In our model, the initial length of a single MT or MF spring is approximately equal to $R-r$, and the initial length of a single IF seems much smaller than those of MT or MF $\left(\ell_{i} \ll \ell_{t} \sim \ell_{a}\right)$. If we assume that the number of three CSK filaments is in the same order of magnitude $\left(n_{t} \sim n_{a} \sim n_{i}\right)$, and note that the cytomembrane is so weak relative to CSK filaments, then we have

$$
K_{\mathrm{m}} \ll \underbrace{K_{a}}_{\frac{h^{2}}{R^{2}} n_{a} k_{a}} \ll \underbrace{K_{t}}_{\frac{h^{2}}{R^{2}} n_{t} k_{t}} \ll \underbrace{K_{i}}_{n_{i} k_{i}} .
$$

Equation (17) indicates that our assumptions about the relative strength of three springs in the derivation of nucleus translocation are reasonable. The above relationship will also allow us to further compare the longitudinal heights of nucleus in three orientations. As shown in Table 3, we found that the sequence order of longitudinal height in three orientations from our model is consistent with those from experimental data (Fig. 1).

\subsection{Distribution of FACs}

Focal adhesions are the main sites of force application through which the cytoskeleton of a cell connects to the ECM for anchoring the cell. Cells placed onto the differently orientated substrate may require distinct forces to maintain their mechanical stability, respectively. Correlation with the lateral size of the FAs showed that there exists the following linear relationship between applied force and area of a single FA (Schwarz et al. 2002). Thus, a cell placed in $U_{-}, D-$, or $E$-orientated substrate, which possesses the angle of $180^{\circ}$, $0^{\circ}$, or $90^{\circ}$ in relating the outer normal of the substrate to the gravity vector, could result in differential FAC size and distribution.

To further reveal the underlying mechanisms, a simple model was developed to understand the orientation dependence of FAs size and distribution. As shown in Fig. 4d, the FA is simplified as an adhesion cluster of parallel bonds with shared force $F$. The adhesion cluster is modeled as a collection of $N_{t}$ molecules near an adhesive surface, in which, at a given time $t$, a number of $N(t)$ molecules are bound and a number of $N_{t}-N(t)$ molecules are unbound. The force $F$ applied to the cluster, which is consists of prestress carried by MFs and additional force carried by weight of nucleus, is assumed to be shared equally by the $N(t)$ closed inactive bonds $\left(0 \leq N(t) \leq N_{t}\right)$. At any given time, each of the bonds can break with a dissociation rate $k_{\text {off }}$ and each of the unbound molecules can form a bond with an association rate $k_{\text {on }}$. Following Bell's model (Bell 1978), dissociation rate is assumed to increase with force $f$ as $k_{\text {off }}=k_{0} \mathrm{e}^{f / F_{b}}$, where the force scale $F_{b}=k_{B} T / x_{b}$ is set by thermal energy $k_{B} T$ and the distance $x_{b}$ between the minimum potential energy and 
Table 4 Stretched length of a single MF and corresponding stress in three orientations

\begin{tabular}{llll}
\hline Orientation & $\Delta \ell_{\mathrm{a}}$ & $F_{g}(\varphi)$ & $f_{g}(\varphi)$ \\
\hline$U$ & $-\theta \frac{G}{K_{\mathrm{t}}}$ & $-G \frac{\theta}{N_{c}} \frac{K_{\mathrm{a}}}{K_{\mathrm{t}}}$ & $-3.75 \times 10^{-4}$ \\
$D$ & $\theta \frac{G}{K_{\mathrm{a}}}$ & $G \frac{\theta}{N_{c}}$ & $7.5 \times 10^{-4}$ \\
$E$ & $\frac{2}{3} \theta^{2} \frac{G}{K_{\mathrm{a}}}(1+\sin \varphi)$ & $\frac{2}{3} G \frac{\theta^{2}}{N_{c}}(1+\sin \varphi)$ & $5.0 \times 10^{-5} \times(1+\sin \varphi)$ \\
\hline
\end{tabular}

the transition state barrier along the reaction coordinate of bond rupture. For typical values $x_{b} \sim 1 \mathrm{~nm}$ and $T \sim 300 \mathrm{~K}$, we find the typical force scale $F_{b} \sim 4 \mathrm{pN}$. Association rate $k_{\text {on }}$ is assumed to be independent of force and taken as a constant (infinite reservoir of ligand).

Conveniently, it is helpful to introduce the dimensionless time $\tau=k_{0} t$, force $f=F / F_{b}$ and the association rate $\gamma=k_{\mathrm{on}} / k_{0}$. The following rate equation predicts the number of formed bonds (Schwarz and Safran 2013):

$\frac{\mathrm{d} N}{\mathrm{~d} \tau}=\gamma\left(N_{t}-N\right)-N \mathrm{e}^{f / N}$

While the first term representing rebinding is linear in the number of bonds, the second term representing forced unbinding is highly nonlinear, therefore leading to interesting feedback effects. Over a sufficiently long time, an equilibrium will be approached such that the right-hand side of Eq. (18) is zero. Thus, we have,

$N=\frac{f N_{t}}{f-N_{t} \operatorname{pln}\left(-\frac{f}{\gamma N_{t}} \mathrm{e}^{f / N_{t}}\right)}$,

where the product logarithm $\operatorname{pln}(a)$ is defined as the solution of $x \mathrm{e}^{x}=a$. A bifurcation analysis of its steady-state behavior shows that the system is unstable (no steady-state solution exists) when the force exceeds a critical value $f_{c}=N_{t} \operatorname{pln}(\gamma / \mathrm{e})$ (Bell 1978). Equation (19) indicates that the tension force acting on talin by microfilament has an effect on the size of FACs.

To simplify our modeling, we assume that multiple transmembrane integrins are bundled together and distributed uniformly on the cell membrane (circled dots in Fig. 4d). The average total integrin density on the cell surface has been estimated as $\rho \sim 1-3 \times 10^{2} \mu^{-2}$ (Wiseman et al. 2004) in either closed inactive or open active conformation. At least in some cells like platelets, more than $95 \%$ of all integrins are in the closed inactive conformation in the absence of ligand (Tadokoro et al. 2003). The results from our previous experiment (Zhang et al. 2017) show that the number of FACs is about 50. Without loss of generality, we consider $N_{c}=50$ clusters distributed uniformly on the membrane, and each cluster contains $N_{t}=300$ effective integrins. They can bind to ligands on the matrix substrate, forming a FAC, to which an
MF is connected. The relative size of a FAC can be described by the fraction of closed inactive bonds $S=N / N_{t} \in[0,1]$. MFs in differently orientated cells or at different polar angles in the same cell may bear different stress, resulting in distinct dissociation rate and eventually causing different size and distribution of FACs. In Eq. (19), force $f$ consists of prestress of MF and additional force carried by weight of nucleus. Force and protein assembly are linearly coupled at FAs, resulting in a constant stress for adhesions of about 5 $\mathrm{nN} / \mathrm{nm}^{2}$ (Balaban et al. 2001). A rough estimate, assuming two-dimensional close packing of integrins, leads to a force in the adhesion site of a few $\mathrm{pN}$ per molecule, similar to that exerted by a single myosin molecule the force. We assume that

$f=\frac{4 \mathrm{pN}+F_{g}(\varphi)}{F_{b}}=1+f_{g}(\varphi)$

where $4 \mathrm{pN}$ comes from prestress of $\mathrm{MF}$ and $F_{g}(\varphi)$ is additional force carried by weight of nucleus. Here we consider the size and distribution of FACs in three orientations, respectively. From the previous discussions about nucleus translocation, we can find the stretched length $\ell_{\mathrm{a}}$ of a single MF and corresponding stress $F_{g}(\varphi)$ in three orientations. Furthermore, we can obtain the numerical value of $f_{g}(\varphi)$ if we assume that $\theta=0.1$ and $K_{\mathrm{a}} / K_{\mathrm{t}}=0.5$ (Table 4 ). We put the value of $f_{g}(\varphi)$ into Eqs (19) and (20) and obtained the FAC size and distribution (Fig. 5).

From Fig. 5, we found that the sequence order of FAC size in three orientations from our model is $E \lesssim D \lesssim U$. The distribution of FAC size is independent of the angle $\varphi$ in $U$ - and $D$-orientation, while it presents a similar inverted sine curve distribution in $E$-orientation. However, there are no statistically significant differences among magnitudes of FAC size in three orientations as the range of longitudinal coordinate is tiny.

\subsection{Equilibrium time}

In above sections, we discussed how much translocation of a nucleus can occur in three orientations under gravity and how this translocation could result in CSK remodeling and FAC reorganization. Here we attempt to estimate how much time the translocation will take before reaching a balance. In 


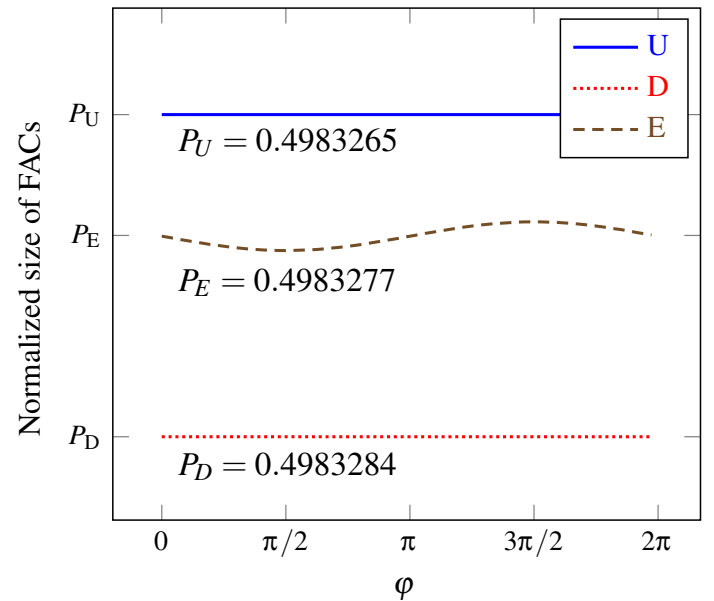

Fig. 5 FAC size distribution at different polar angles ( $c f$. Fig. 4) in three orientations. FAC size is independent of the angle in $U$ - and $D$ orientation, while it presents an inverted sine curve-like distribution in E-orientation

each of the above three kinds of nucleus translocation, the mechanical equilibrium equation for nucleus can be rewritten as

$G=K \Delta h$,

where $K$ is equivalent spring constant and $K=K_{i}, K_{a}$, $K_{a} R / h$ for $U-, D-, E$-orientation, respectively. Thus, nucleus movements can be described by the following second-order differential equation,

$\frac{\mathrm{d}^{2} h}{\mathrm{~d} t^{2}}=\frac{G}{m}-\frac{K}{m} h-\frac{\gamma}{m} \frac{\mathrm{d} h}{\mathrm{~d} t}=\frac{G}{m}-\omega^{2} h-2 \zeta \omega \frac{\mathrm{d} h}{\mathrm{~d} t}$,

where $\gamma$ is the damping coefficient which describes the friction caused by viscous drag force between cytosol and cytoskeleton and $m$ is the mass of the nucleus. $\omega=\sqrt{K / m}$ is called the (undamped) natural frequency of the system, and $\zeta=\gamma / 2 \sqrt{\mathrm{Km}}$ is called the damping ratio. The behavior of the system depends on the relative values of the two fundamental parameters, the natural frequency $\omega$ and the damping ratio $\zeta$. As shown in Fig. 6, the qualitative behavior of the system depends crucially on whether the quadratic equation for $\zeta$ has one real solution $(\zeta=1$, critical damping), two real solutions $(\zeta>1$, overdamping), or two complex conjugate solutions $(0 \leq \zeta<1$, underdamping).

The nucleus translocation is an overdamped process, since the motions of the cytoskeleton and cells are overdamped (Howard 2001). It can be seen that overdamped system takes an extremely long time (theoretically infinite time) to return to its static equilibrium position. When the distance of the nucleus translocation from its static equilibrium position equal to or less than $\sqrt{2 k_{B} T / K}$, we assume that the system will reach the equilibrium, since in this case the gravitational

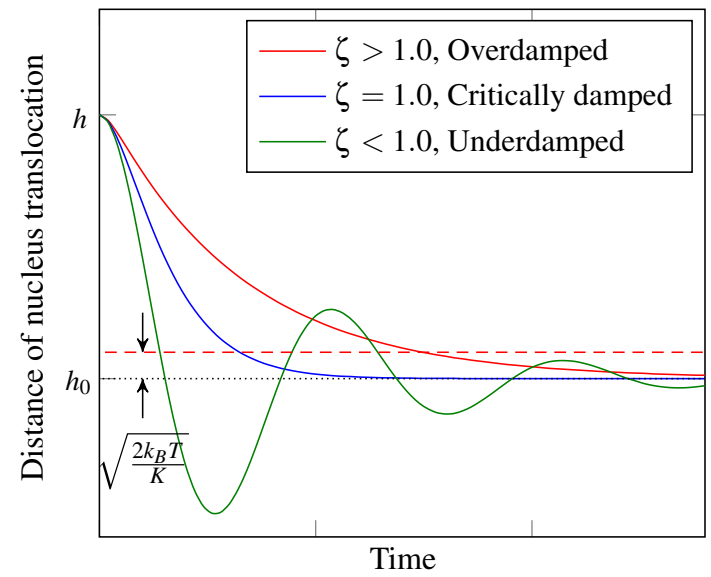

Fig. 6 Estimation of characteristic equilibrium time of nucleus sedimentation in damped cytosol. Time dependence of the system behavior is calculated upon Eq. (22) and presented on the value of the damping ratio $\zeta$, for underdamped, critically damped, and overdamped cases, at zero-velocity initial condition. $h_{0}$ is the balance height

potential energy of the nucleus is less than $k_{B} T$. Thus, if damping coefficient $\gamma$ and equivalent spring constant $K$ are given, we could estimate the time needed to reach equilibrium. Unfortunately, it is impossible to estimate the value of $\gamma$ and $K$ from our data in hand.

\section{Discussions and conclusion}

Translocation of the dense nucleus along the gravity initiates mechanical remodeling in a mammalian cell. By simplifying the cell as a nucleated tensegrity structure, we proposed a theoretical biomechanical model to integrate the mechanosensing of nucleus translocation with CSK remodeling and FAC reorganization induced by a gravity vector in upward, downward, or edge-on oriented substrate. In our model, the cell and CSK filaments are considered to be symmetrical, and all elements for supporting nucleus, including CSK filaments and cytomembrane, are simplified as springs. The FA is simplified as an adhesion cluster of parallel bonds with shared force.

Our model shows that dense nucleus tends to translocate under gravity. This translocation will exert additional force on cytoskeleton and compress or stretch CSK filaments. By remodeling, cytoskeleton changes its stress or tension and generates a corresponding reaction force on nucleus to achieve an equilibrium. Finally, changes of the tension force acting on talin through microfilament will bring up changes in the size of FACs. Results from our model are in qualitative agreement with those from experiments. Specifically, we have found that the translocation of nucleus is higher in $D$ compared with those in $U$ or $E$. FAC size is independent with the angle $\varphi$ in $U$ - and $D$-orientation, while it presents a 
similar inverted sine curve distribution in $E$-orientation. The FAC size magnitudes of cells in three orientated substrate were found no statistically significant differences. Additionally, our model presents a possible method to theoretically estimate characteristic equilibrium time of nucleus sedimentation, although it is not feasible at present.

As mentioned previously, there are still remarkable differences between our works on normally sized mammalian cells (Zhang et al. 2017 and the current work) and those on large X. laevis oocytes (Feric and Brangwynne 2013; Feric et al. 2015). First, while those super-sized $X$. laevis oocytes serve as a good model system to elucidate the impacts of gravity vector, our current work focuses on understanding this process for a normally sized mammalian cell that is universal and realistic for the community for mammals. Second, the underlying mechanisms in gravisensation are quite different, where the former is to understand the sedimentation and aggregation of the nucleolus inside the nucleus, but the latter is to elucidate the translocation of the entire nucleus inside the cytosol. Both models have different viscoelastic properties for translocating the nucleolus or the nucleus. Third, as stated in our previous paper (Zhang et al. 2017), the impact of the gravity for a normally sized mammalian cell (in the order of $0.5 \mathrm{pN}$ ) on directing cell mechanosensing could not be neglected mainly due to the integral effect of dense organelle sedimentation. Thus, quantifying the nuclear translocation, positioning, and characteristic sedimentation time from our modeling is meaningful to understand the dynamics of nucleus movement inside the cytosol, which is hard to determine experimentally. We have added these into the Discussion section.

Major assumptions of our modeling satisfy the key characteristics of a real adherent cell. For example, the symmetry of cell morphology and CSK distribution is reasonably simplified because an isolated cell is fully spread without polarity and the majority of microfilaments and microtubules is radially organized (Vorselen et al. 2014; Zhang et al. 2017). Meanwhile, FACs are mainly composed of equally weighted, multiple integrin-ligand bonds (Schwarz and Safran 2013). Our model is a simplified or idealized one. However, it is still meaningful for understanding the mechanical remodeling of a normally sized mammalian cell under vector-directed gravity, which is a critical issue for mammalian gravisensation, but poorly understood yet. This also makes sense, especially when matching the predictions from this model well with our experiments previously described. Of course, like most of the existing models, there are several limitations of this model. The first comes from the symmetrical assumption of cell shape and CSK distribution. In reality, not all of those isolated cells present the symmetrical shapes and cytoskeletons, but their asymmetry is usually averaged out when a large number of cells are lumped together. The second is attributed to the decoupling of the three CSK components, which might bias their effects on nucleus translocation. Noticing that the CSK network is quite complicated, no universal models are available to fully describe the intrinsic characteristics of the cytoskeleton, and thus, it is necessary to simplify the cytoskeletons for a specific issue. The third derives from the fact that the parameters used in the model are hard to be determined by experiments. Fortunately, most of the results and predictions from our modeling are less sensitive to the values of those parameters.

Acknowledgements This work was supported by Strategic Priority Research Program of Chinese Science Academy of Sciences grant XDA04020219, National Natural Science Foundation of China grant 31110103918, and National Key Basic Research Foundation of China grant $2011 \mathrm{CB} 710904$.

\section{Compliance with ethical standards}

Conflict of interest The authors declare that they have no conflict of interest.

\section{Appendix 1: Length changes of MT or MF in $E$}

As shown in Fig. 7, we set up a three-dimensional Cartesian coordinate system by taking the $y$-axis in the opposite direction of gravity and $x-y$ coordinate plane at the contact surfaces between cell and substrate. $R$ is cell radius, $r$ is nucleus radius, and $h$ is the distance from nucleus centroid to the substrate surface. $\theta$ is the angle between MFs or MTs and substrates. $\overline{p^{P}}$ is a radial MT/MF, and $\varphi$ is the angle between the projections of $\overline{p^{P}}$ on $x-y$ coordinate plane and $x$ axis.

Cartesian coordinate for points $P$ and $p$ can be determined from the coordinate transformations

$P=(R \cos \varphi, R \sin \varphi, 0), \quad p=(r \cos \varphi, r \sin \varphi, h)$.

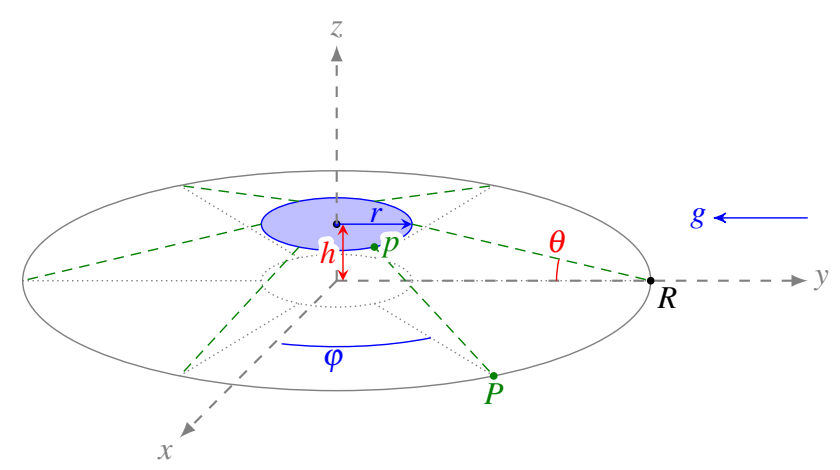

Fig. 7 A nucleated cell in $E$-oriented substrate in the threedimensional coordinate system before nucleus translocation 
Then, the initial length of MFs and MTs can be expressed as

$$
\begin{aligned}
\ell_{0} & =\sqrt{(R \cos \varphi-r \cos \varphi)^{2}+(R \sin \varphi-r \sin \varphi)^{2}+h^{2}} \\
& =\sqrt{(R-r)^{2}+h^{2}} .
\end{aligned}
$$

\section{If $\Delta h^{E}=\Delta h_{\|}$, the compressed length and additional force of MTs}

If nucleus translocation $\Delta h^{E}=\Delta h_{\|}$is parallel to gravity, Cartesian coordinate for point $p$ will be changed into:

$p^{\prime}=\left(r \cos \varphi, r \sin \varphi-\Delta h_{\|}, h\right)$.

The corresponding length of MTs can be expressed as:

$$
\begin{aligned}
\ell\left(\Delta h_{\|}\right)= & \frac{\sqrt{(R \cos \varphi-r \cos \varphi)^{2}+(R \sin \varphi-r \sin \varphi+}}{\left.+\Delta h_{\|}\right)^{2}+(0-h)^{2}} \\
= & \sqrt{(R-r)^{2}+h^{2}+\Delta h_{\|}^{2}+2(R-r) \Delta h_{\|} \sin \varphi} \\
= & \sqrt{\ell_{0}^{2}+\Delta h_{\|}^{2}+2(R-r) \Delta h_{\|} \sin \varphi} .
\end{aligned}
$$

Then, the Taylor polynomial for $\ell\left(\Delta h_{\|}\right)$at zero is defined by

$\ell\left(\Delta h_{\|}\right)=\ell_{0}+\frac{(R-r) \sin \varphi}{\ell_{0}} \Delta h_{\|}+O\left(\Delta h_{\|}^{2}\right)$

Thus, we have

$\Delta \ell=\ell-\ell_{0} \approx \frac{(R-r) \sin \varphi}{\ell_{0}} \Delta h_{\|}=\sin \varphi \cos \theta \Delta h_{\|}$,

where $\varphi \in(\pi, 2 \pi)$ since MTs mainly resist compression. We can find the $y$ component resultant force $F_{y}$ by the algebraic summing of the components of the forces in the $y$ direction,

$$
\begin{aligned}
F_{y} & =-\int_{\pi}^{2 \pi} \frac{n_{t} k_{t}}{2 \pi} \sin \varphi \cos \theta \Delta h_{\|} \cos \theta \mathrm{d} \varphi \\
& =-\frac{n_{t} k_{t}}{2 \pi} \Delta h_{\|} \cos ^{2} \theta \int_{\pi}^{2 \pi} \sin \varphi \mathrm{d} \varphi \\
& =\frac{n_{t} k_{t}}{\pi} \Delta h_{\|} \cos ^{2} \theta \\
& \approx \frac{n_{t} k_{t}}{\pi} \Delta h_{\|} .
\end{aligned}
$$

\section{If $\Delta h^{E}=\Delta h_{\perp}$, the stretched length and additional}

\section{force of MFs}

If nucleus migration $\Delta h^{E}=\Delta h_{\perp}$ is perpendicular to MT, Cartesian coordinate for point $p$ will be changed into

$p^{\prime}=\left(r \cos \varphi, r \sin \varphi-\Delta h_{\perp} \sin \theta, h+\Delta h_{\perp} \cos \theta\right)$.
The corresponding length of MFs can be expressed as

$$
\begin{aligned}
\ell\left(\Delta h_{\perp}\right)= & \frac{\sqrt{(R \cos \varphi-r \cos \varphi)^{2}+(R \sin \varphi-r \sin \varphi+}}{\left.+\Delta h_{\perp} \sin \theta\right)^{2}+\left(h+\Delta h_{\perp} \cos \theta\right)^{2}} \\
= & \frac{\sqrt{(R-r)^{2}+2(R-r) \Delta h_{\perp} \sin \varphi \sin \theta+}}{+h^{2}+\Delta h_{\perp}^{2}+2 h \Delta h_{\perp} \cos \theta} \\
= & \frac{\sqrt{2[(R-r) \sin \varphi \sin \theta+h \cos \theta] \Delta h_{\perp}+}}{+\ell_{0}+\Delta h_{\perp}^{2}} .
\end{aligned}
$$

Then, the Taylor polynomial for $\ell\left(\Delta h_{\perp}\right)$ at zero is defined by

$$
\begin{aligned}
\ell\left(\Delta h_{\perp}\right)=\ell_{0}^{2} & +\frac{(R-r) \sin \varphi \sin \theta+h \cos \theta}{\ell_{0}} \Delta h_{\perp} \\
& +O\left(\Delta h_{\perp}^{2}\right) .
\end{aligned}
$$

Thus, we have

$$
\begin{aligned}
\Delta \ell & =\ell-\ell_{0} \approx \frac{(R-r) \sin \varphi \sin \theta+h \cos \theta}{\ell_{0}} \Delta h_{\perp} \\
& =\sin \theta \cos \theta(1+\sin \varphi) \Delta h_{\perp} .
\end{aligned}
$$

We can find $y$ and $z$ components resultant force $F_{y}$ and $F_{z}$ by the algebraic summing of the components of the forces in $y$ and $z$ directions,

$$
\begin{aligned}
F_{y} & =\int_{0}^{2 \pi} \frac{n_{a} k_{a}}{2 \pi} \sin \theta \cos \theta(1+\sin \varphi) \Delta h_{\perp} \cos \theta \sin \varphi \mathrm{d} \varphi \\
& =\frac{n_{a} k_{a}}{2 \pi} \Delta h_{\perp} \sin \theta \cos ^{2} \theta \int_{0}^{2 \pi}(1+\sin \varphi) \sin \varphi \mathrm{d} \varphi \\
& =\frac{n_{a} k_{a}}{2} \Delta h_{\perp} \sin \theta \cos ^{2} \theta \\
& \approx \frac{1}{2} n_{a} k_{a} \theta \Delta h_{\perp} \\
F_{z} & =\int_{0}^{2 \pi} \frac{n_{a} k_{a}}{2 \pi} \sin \theta \cos \theta(1+\sin \varphi) \Delta h_{\perp} \sin \theta \mathrm{d} \varphi \\
& =\frac{n_{a} k_{a}}{2 \pi} \sin ^{2} \theta \cos \theta \Delta h_{\perp} \int_{0}^{2 \pi}(1+\sin \varphi) \mathrm{d} \varphi \\
& =n_{a} k_{a} \sin ^{2} \theta \cos \theta \Delta h_{\perp} \\
& \approx n_{a} k_{a} \theta^{2} \Delta h_{\perp}
\end{aligned}
$$

\section{References}

Balaban NQ, Schwarz US, Riveline D, Goichberg P, Tzur G, Sabanay I, Mahalu D, Safran S, Bershadsky A, Addadi L et al (2001) Force and focal adhesion assembly: a close relationship studied using elastic micropatterned substrates. Nat Cell Biol 3(5):466 
Bell GI (1978) Models for the specific adhesion of cells to cells. Science 200(4342):618

Clément G, Slenzka K (2006) Fundamentals of space biology: research on cells, animals, and plants in space, vol 18. Springer, New York

Cogoli A, Cogoli-Greuter M (1996) Activation and proliferation of lymphocytes and other mammalian cells in microgravity. Adv Space Biol Med 6:33

Feric M, Brangwynne CP (2013) A nuclear F-actin scaffold stabilizes RNP droplets against gravity in large cells. Nat Cell Biol 15(10): 1253

Feric M, Broedersz CP, Brangwynne CP (2015) Scientific reports 5

Fletcher DA, Mullins RD (2010) Cell mechanics and the cytoskeleton. Nature 463(7280):485

Geiger B, Spatz JP, Bershadsky AD (2009) Environmental sensing through focal adhesions. Nat Rev Mol Cell Biol 10(1):21

George TF, Jelski D, Letfullin RR, Zhang G (2011) Computational studies of new materials II: from ultrafast processes and nanostructures to optoelectronics, energy storage and nanomedicine. World Scientific, Singapore

Grimm D, Wise P, Lebert M, Richter P, Baatout S (2011) How and why does the proteome respond to microgravity? Expert Rev Proteom 8(1):13

Herrmann H, Bär H, Kreplak L, Strelkov SV, Aebi U (2007) Intermediate filaments: from cell architecture to nanomechanics. Nat Rev Mol Cell Biol 8(7):562

Howard J et al (2001) Mechanics of motor proteins and the cytoskeleton. Sinauer Associates, Sunderland

Ingber DE (1993) Cellular tensegrity: defining new rules of biological design that govern the cytoskeleton. J Cell Sci 104(3):613

Kalwarczyk T, Ziebacz N, Bielejewska A, Zaboklicka E, Koynov K, Szymanski J, Wilk A, Patkowski A, Gapinski J, Butt HJ et al (2011) Comparative analysis of viscosity of complex liquids and cytoplasm of mammalian cells at the nanoscale. Nano Lett 11(5):2157

Kamei H (1994) Relationship of nuclear imaginations to perinuclear rings composed of intermediate filaments in MIA PaCa- 2 and some other cells. Cell Struct Funct 19(3):123

Li H, Chen J, Zhang Y, Sun S, Tao Z, Long M (2010) Effects of oriented substrates on cell morphology, the cell cycle, and the cytoskeleton in Ros 17/2.8 cells. Sci China Life Sci 53(9):1085

Lim C, Zhou E, Quek S (2006) Mechanical models for living cells-a review. J Biomech 39(2):195

Milo R, Phillips R (2015) Cell biology by the numbers. Garland Science, New York

Mofrad MR, Kamm MR (2006) Cytoskeletal mechanics: models and measurements in cell mechanics. Cambridge University Press, Cambridge
Nava MM, Raimondi MT, Pietrabissa R (2014) Bio-chemo-mechanical models for nuclear deformation in adherent eukaryotic cells. Biomech Model Mechanobiol 13(5):929

Nichols HL, Zhang N, Wen X (2006) Proteomics and genomics of microgravity. Physiol Genom 26(3):163

Orr AW, Helmke BP, Blackman BR, Schwartz MA (2006) Mechanisms of mechanotransduction. Dev Cell 10(1):11

Pollard EC (1965) Theoretical studies on living systems in the absence of mechanical stress. J Theor Biol 8(1):113

Prescott D, Myerson D, Wallace J (1972) Enucleation of mammalian cells with cytochalasin B. Exp Cell Res 71(2):480

Satcher R, Dewey CF (1996) Theoretical estimates of mechanical properties of the endothelial cell cytoskeleton. Biophys J 71(1):109

Schwarz US, Safran SA (2013) Physics of adherent cells. Rev Mod Phys 85(3):1327

Schwarz US, Balaban NQ, Riveline D, Bershadsky A, Geiger B, Safran S (2002) Calculation of forces at focal adhesions from elastic substrate data: the effect of localized force and the need for regularization. Biophys J 83(3):1380

Stamenović D, Coughlin MF (1999) The role of prestress and architecture of the cytoskeleton and deformability of cytoskeletal filaments in mechanics of adherent cells: a quantitative analysis. J Theor Biol 201(1):63

Tadokoro S, Shattil SJ, Eto K, Tai V, Liddington RC, de Pereda JM, Ginsberg MH, Calderwood DA (2003) Talin binding to integrin $B$ tails: a final common step in integrin activation. Science 302(5642): 103

Vorselen D, Roos WH, MacKintosh FC, Wuite GJ, van Loon JJ (2014) The role of the cytoskeleton in sensing changes in gravity by nonspecialized cells. FASEB J 28(2):536

Wang N, Tytell JD, Ingber DE (2009) Mechanotransduction at a distance: mechanically coupling the extracellular matrix with the nucleus. Nat Rev Mol Cell Biol 10(1):75

Wegener J, Janshoff A, Galla HJ (1998) Cell adhesion monitoring using a quartz crystal microbalance: comparative analysis of different mammalian cell lines. Eur Biophys J 28(1):26

Wiseman PW, Brown CM, Webb DJ, Hebert B, Johnson NL, Squier JA, Ellisman MH, Horwitz A (2004) Spatial mapping of integrin interactions and dynamics during cell migration by image correlation microscopy. J Cell Sci 117(23):5521

Zhang C, Zhou L, Zhang F, Lü D, Li N, Zheng L, Xu Y, Li Z, Sun S, Long M (2017) Mechanical remodeling of normally sized mammalian cells under a gravity vector. FASEB J 31(2):802 